\title{
The onset site of rheumatoid arthritis: the joints or the lung?
}

\author{
G. Cafaro, A. Alunno, V. Valentini, M.C. Leone, \\ E. Marcucci, E. Bartoloni, R. Gerli \\ Rheumatology Unit, Department of Medicine, University of Perugia, Italy
}

\begin{abstract}
SUMMARY
The etiopathogenesis of rheumatoid arthritis (RA) is not yet fully elucidated and the site of inflammation onset is still a matter of debate. The presence of autoantibodies as well as clinical manifestations, such as interstitial lung disease, before the onset of arthritis seems to be in favour of the hypothesis that initial pathogenic events take place in tissues other than the joint. In this review article we summarize the most recent literature on extra-synovial autoimmunity triggers eventually leading to RA, with particular focus on the role of the lung. To date, anti-cyclic citrullinated peptide antibodies (ACPAs) are considered central players in RA pathogenesis and represent the gold-standard for disease diagnosis. Lungs and mucosae are exposed to environmental stimuli such as dusts and smoke which have been shown to foster citrullination of peptides in lungs thereby triggering the production of ACPA. In addition, other mechanisms of disease pathogenesis independent of citrullination play an important role. Deeper knowledge of these processes could represent a huge step forward in the management of RA, with dramatic impact on diagnosis, prevention, prognostic stratification and treatment of the disease.
\end{abstract}

Key words: Rheumatoid arthritis; lung; ACPA; smoking.

Reumatismo, 2016; 68 (4): 167-175

\section{INTRODUCTION}

heumatoid arthritis (RA) is a systemic disease characterized by chronic articular inflammation. The main clinical features of RA are joint swelling, tenderness and progressive destruction, culminating with loss of articular function, disability and premature mortality. Although in the majority of patients RA is limited to joint involvement, a consistent proportion of subjects develops extra-articular manifestations (EAMs) during the course of the disease. EAMs can virtually affect any organs and tissues and severely worsen patients' quality of life as well as disease prognosis and, in fact, they are associated with increased morbidity and mortality in RA patients. Among EAMs, the involvement of the respiratory system, namely airways, pleurae and pulmonary parenchyma, is rather common and represents the second cause of death in patients with RA after cardiovascular events. The reported prevalence widely varies among studies ranging from 4 to $50 \%$ (1). This is due, at least in part, to the heterogeneity of the clinical picture and misdiagnosis in patients with no or very mild symptoms.

In recent years, a growing number of studies has suggested that the initiation of the disease may occur in sites different from the joints, such as the bone marrow (2), the gastrointestinal apparatus or the lung. Among these, the latter may represent a key aspect in the pathogenesis of the disease. In particular, the hypothesis been put forward that the trigger event leading to disease development may occur in the lung rather than in the joints, as previously suggested. Following this lead, we aim here to provide an overview on recent insights regarding RA-associated pulmonary involvement and its possible relationship with disease pathogenesis.

\section{RHEUMATOID ARTHRITIS- ASSOCIATED INTERSTITIAL LUNG DISEASE}

The clinical spectrum of respiratory diseases associated with RA is rather hetero-
Corresponding author Roberto Gerli

Rheumatology Unit, Department of Medicine University of Perugia Via Enrico dal Pozzo 06100 Perugia, Italy E-mail: roberto.gerli@unipg.it 
geneous and includes several distinct conditions affecting airways (bronchiectasis, bronchiolitis), pleurae (pleuritis, pleural effusion), blood vessels (pulmonary arterial hypertension, pulmonary vasculitis), lung parenchyma [rheumatoid nodules, infections and interstitial lung disease (ILD)]. The overall prevalence of ILD in RA ranges from 7 to $50 \%$ and the most common radiological pattern, as proven by high resolution computed tomography (HRCT), is the usual interstitial pneumonia (UIP) $(65 \%)(3-5)$.

UIP is characterized by peripheral, subpleural, basal, reticulation and honeycombing as well as traction bronchiectasis and architectural distortion. The second most frequent RA-ILD pattern is nonspecific interstitial pneumonia (NSIP) (24\%) which appears as bilateral, symmetrical, patchy, mainly basal ground-glass areas with little or no honeycombing on HRCT. Less frequent patterns include organizing pneumonia (OP) (5\%), acute interstitial pneumonia, desquamative interstitial pneumonia and lymphoid interstitial pneumonia (6-9). Symptoms complained of by RA patients with ILD include various degrees of exertional dyspnoea and coughing that require further investigation with pulmonary function tests (PFT), chest X-ray and eventually HRCT. However, the diagnosis of RA-ILD may often be incidental as $30-90 \%$ of patients with RA-ILD, according to different studies, may have no or very mild respiratory symptoms $(10,11)$. The burden of clinically manifest ILD on RA prognosis is critical, with an overall median survival ranging between 2.6 and 8.1 years after diagnosis. The most important factors contributing to a worse prognosis are UIP pattern, with a poor prognosis similar to that of idiopathic pulmonary fibrosis (IPF), older age, male gender and lower diffusing capacity of the lung for carbon monoxide (DLCO) $(12,13)$. In patients with RA-ILD, DLCO is often reduced and may be used not only for diagnosis but also for monitoring disease evolution, together with PFT, six-minute walking test and, when required, HRCT.

Although RA-ILD is doubtless one of the most clinically relevant features of respiratory EAMs in RA, airway abnormalities, often totally asymptomatic, seem to be even more common. They become evident earlier in the history of the disease, mainly as bronchial dilatation that may evolve into wall thickening, acquiring the features of bronchiolitis with the progression of the disease (14).

\section{RISK FACTORS}

Several genetic, demographic and environmental factors have been associated with an increased risk of developing ILD in patients with RA. The human leukocyte antigen (HLA)-DRB $1 * 04$, containing a preserved sequence of amino acids in the hypervariable region, known as shared epitope, is associated with an increased risk of developing RA (15). Surprisingly, this haplotype appears to be protective against ILD [odds ratio $(\mathrm{OR})=0.66$ ]. This observation comes from a Japanese study that evaluated 450 patients and therefore needs to be confirmed with larger cohorts of patients including also other ethnic groups (16). In addition, HLA-DQB $1 * 04$ appeared to be protective against ILD $(\mathrm{OR}=0.55)$, while HLA-DRB $1 * 15$ and 16 and DQB $1 * 06$ were seemingly associated with an increased risk for ILD (ORs=15.21, 1.75 and 1.57 respectively) (16).

Demographic characteristics, such as male gender and older age at onset, have now been confirmed to be associated with ILD by several studies $(9,10,13,17)$.

As far as environmental factors are concerned, tobacco smoking has also been associated with the risk of developing both RA and lung disease, the latter with an OR ranging from 1.91 to $3.8(7,9,18)$. Tobacco exposure has also been suggested as one of the possible initiating factors of the disease. Furthermore, the risk of RA development due to smoking is increased in patients also exposed to silica dust (19). In this context, since silica dust enters the body through the respiratory system as happens with smoke, it may be speculated that silica acts synergistically with smoke in triggering crucial events of RA pathogenesis. Al- 
though interesting, this observation needs to be further confirmed by additional studies. A growing body of evidence also supports a role of the intestinal mucosal immune system (such as Peyer's patches and mucosa-associated lymphoid tissue) as an initiation site of autoimmunity, both with cell-mediated and humoral mechanisms. Commensal microbiome balance and its alterations (dysbiosis), in the context of a predisposing genetic environment, seems to have a critical function in triggering the autoimmune process (20).

Although their role is still matter of debate, the potential noxious effect of disease-modifying anti-rheumatic drugs (DMARDs) on ILD, including RA-ILD, is worth mentioning. In a recent systematic literature review, Roubille et al. have tried to estimate the prevalence of this deleterious potential adverse event, which may be caused by both conventional and biotechnological DMARDs. However, the results are not conclusive. In most cases, in fact, it is almost impossible to establish whether the development of ILD represents an adverse event linked to the drug or an EAM of the disease, since clinical, radiological and histopathological findings often overlap (21).

Regarding the serological status, several studies reported an association of RA-ILD and airway abnormalities with rheumatoid factor and anti-cyclic citrullinated peptides antibodies (ACPA) positivity $(3,5,9,17$, 22-24). Interestingly, this association resulted independent of other predisposing factors such as male gender and older age, revealing that serological status may be the most important predictor of developing ILD in RA patients. In addition, the work by Giles et al. (3) was able to identify a correlation between ILD severity and the number of ACPAs directed towards different citrullinated peptides, such as fibrinogen peptides, enolase, vimentin and histones. On the other hand, another study failed to confirm the association between ACPA positivity and RA-ILD (25), although it was performed on a very small number of patients. Moreover, a recent meta-analysis reported that RA-ILD is not significantly associated with ACPA in a specific ethnic group, namely a subset of Asian RA patients (23).

Since RF/ACPA positivity is associated with more severe articular disease, the fact that joint disease severity appears to be linked to RA-ILD is easy to explain (10, 13).

In the last few years, additional possible risk factors for ILD have been identified in patients with RA and some of them may have a prominent pathogenic role. Among these, serum levels of matrix metalloproteinase (MMP)-7 and interferon (IFN)- $\gamma$ induced chemokine CXCL10 seem to be fairly specific for RA-ILD (26). The IFNinducible protein 16 (IFI16) was found to be increased in sera of patients with RA compared to healthy controls and IFI16 positivity was associated with the presence of ILD in RA patients independently of smoking and male gender (27). Moreover, newly identified citrullinated heat shock protein (Hsp) 90 may have an important role in the pathogenesis of RA-ILD, as antibodies directed against this target seem to be very specific for lung disease in RA patients compared to IPF and mixed connective tissue disease (MCTD). In particular, anti-citrullinated-Hsp90 antibodies have a specificity of at least $96 \%$ for RA-ILD, since very few patients with RA but not ILD, IPF-ILD or MCTD-ILD display such autoantibodies in the serum (28). Interestingly, the presence of these autoantibodies has also been demonstrated in RA bronchoalveolar lavage (BAL) independently of serum concentration evaluated on matched samples. This observation may further support the hypothesis that the triggering of pathogenetic events of RA may occur in the lung (29).

\section{THE ROLE OF THE LUNG IN RHEUMATOID ARTHRITIS PATHOGENESIS}

It is now well established that serum autoantibodies found in RA patients can be detected years before disease onset. During pre-clinical phases of the disease, the pattern of serum autoantibodies progressively 
changes. In particular, autoantibodies develop cross-reactivity towards an increasing number of epitopes (epitope spreading), increase their avidity and concentration and undergo isotype switch and glycosylation. Among these autoantibodies, ACPA, which are directed towards different citrullinated peptides and epitopes, are the most widely studied and those representing the diagnostic gold standard for RA. The demonstration of such autoantibodies in the serum of subjects without evidence of joint involvement, together with the evidence of airway abnormalities on HRCT scans of patients with circulating RA-related antibodies in the absence of arthritis (30), led to the hypothesis that the immunological process, which eventually evolves to joint inflammation, occurs in other sites of the body (31). In addition, the observation that some RA patients develop ILD prior to joint inflammation further supported the hypothesis that the lung may represent the initiating site of autoimmunity (9).

\section{CITRULLINATION}

Citrullination is a post-translational modification catalyzed by the calcium-dependent enzyme peptidyl-arginine deaminase (PAD), which converts arginine residues into citrulline. This reaction produces epigenetic changes on peptides eventually leading to the regulation of gene transcription and it normally occurs in physiological processes including skin keratinization. However, an increased expression of PAD genes may lead to a larger proportion of citrullinated peptides in tissues, able to initiate autoimmune responses in genetically predisposed subjects. To date, five isoforms of PAD with differential tissue distribution have been identified. PAD 1 and 3 are mainly expressed in the skin, PAD 2 in the central nervous system and hematopoietic cells, PAD 4 and 6 in hematopoietic cells (32). Giles et al. (4) identified crossreactive serum antibodies against PAD 3 and PAD 4 in RA patients and linked their presence to increased levels of citrullination. These antibodies appear to be able to increase the sensitivity of PAD 4 to calci- um and consequently its catalytic capacity. If RA is considered a disease caused by environmental factors acting on genetically predisposed individuals, it is reasonable to hypothesize that triggering events occur in tissues in contact with external agents, such as respiratory and digestive systems. The results of a study published by Willis et al. (33) appear to support the hypothesis of the lung as a site of disease initiation. The authors enrolled two groups of subjects, the first including seronegative healthy controls considered at risk of developing RA (subjects with one $1^{\text {st }}$ degree relative affected) and the second one including patients with seropositive RA. ACPA were detected in the sputum of subjects of the first group, but higher sputum levels of ACPA, compared to the serum, were observed in samples taken from patients of the second group.

As already mentioned, tobacco smoke has been associated with both RA and RA-ILD. Furthermore, it has been demonstrated that tobacco smoke can enhance the expression of the PAD2 enzyme in bronchial mucosa and this may contribute to increasing the amount of citrullinated peptides present in the BAL (34). Smoking has been associated with an increased risk of developing ACPA-positive RA in subjects with HLA-DRB1 SE genotype (35, 36). Conversely, some authors speculated that chronic inflammation, such as occurs in chronic obstructive pulmonary disease, rather than smoking itself, may represent the basis of increased ACPA production in the lungs of smokers and, therefore, of RA development (37). This phenomenon may be due to the development of bronchus-associated lymphoid tissue (BALT) induced by chronic inflammation caused by cigarette smoking (38). Therefore, induced BALT may represent an area where the autoimmune process originates. The hypothesis of chronic mucosal inflammation as a potential trigger of RA autoimmunity appears to be supported by the finding of increased prevalence of $\mathrm{IgG}$ and IgA anti-CCP, IgM and IgA RF antibodies, independently of smoking, sex and age, in patients with bronchiectasis and cystic fi- 
brosis, compared to healthy controls. Furthermore, patients with bronchiectasis and double antibody positivity seem to have a higher rate of RA development compared to healthy controls $(39,40)$. Citrullinated peptides may subsequently be exposed to immune cells either as a consequence of apoptosis or following their release by activated neutrophils within neutrophil extracellular traps (NET) in a process known as NETosis. NETosis is normally initiated in response to microbial infection to activate immune cells and induce the elimination of pathogens, but, in this scenario, it may lead to the production of ACPA (31).

PAD enzyme is produced not only by human cells but also by bacteria such as Porphyromonas gingivalis, normally present in the oral cavity and intestinal tract of healthy individuals. This bacterium has been linked to the development of periodontitis and is able to produce an active form of PAD which can induce peptide citrullination (41).

Taken as a whole, the currently available evidence strongly supports the hypothesis that the autoimmune process in RA may arise in the lung. Therefore, it is crucial to clarify how this process can spread to the joints and lead to chronic synovial inflammation. Several mechanisms have been suggested to find the link between lung and joint inflammation (Figure 1), although they need to be fully elucidated:

1. molecular mimicry: it has been demonstrated that lung and joints share common citrullinated proteins such as vimentin peptides. Therefore, it could be speculated that ACPA produced in extra-articular tissues can subsequently reach target tissues and trigger inflammation (42).

2. Epitope spreading: one study reported that in the pre-clinical phase of RA there is a progressive differentiation of the ACPA pool towards an increasing number of citrullinated epitopes. Therefore, ACPA arising in one tissue may be directed to citrullinated epitopes present in other tissues (43).

3. T-cell migration to the joints: not only B-cells and plasma cells, but also Tcells could have a role in this scenario, migrating from the site of autoimmunity triggering (e.g. the lung) to peripheral tissues, including joints, and subse-

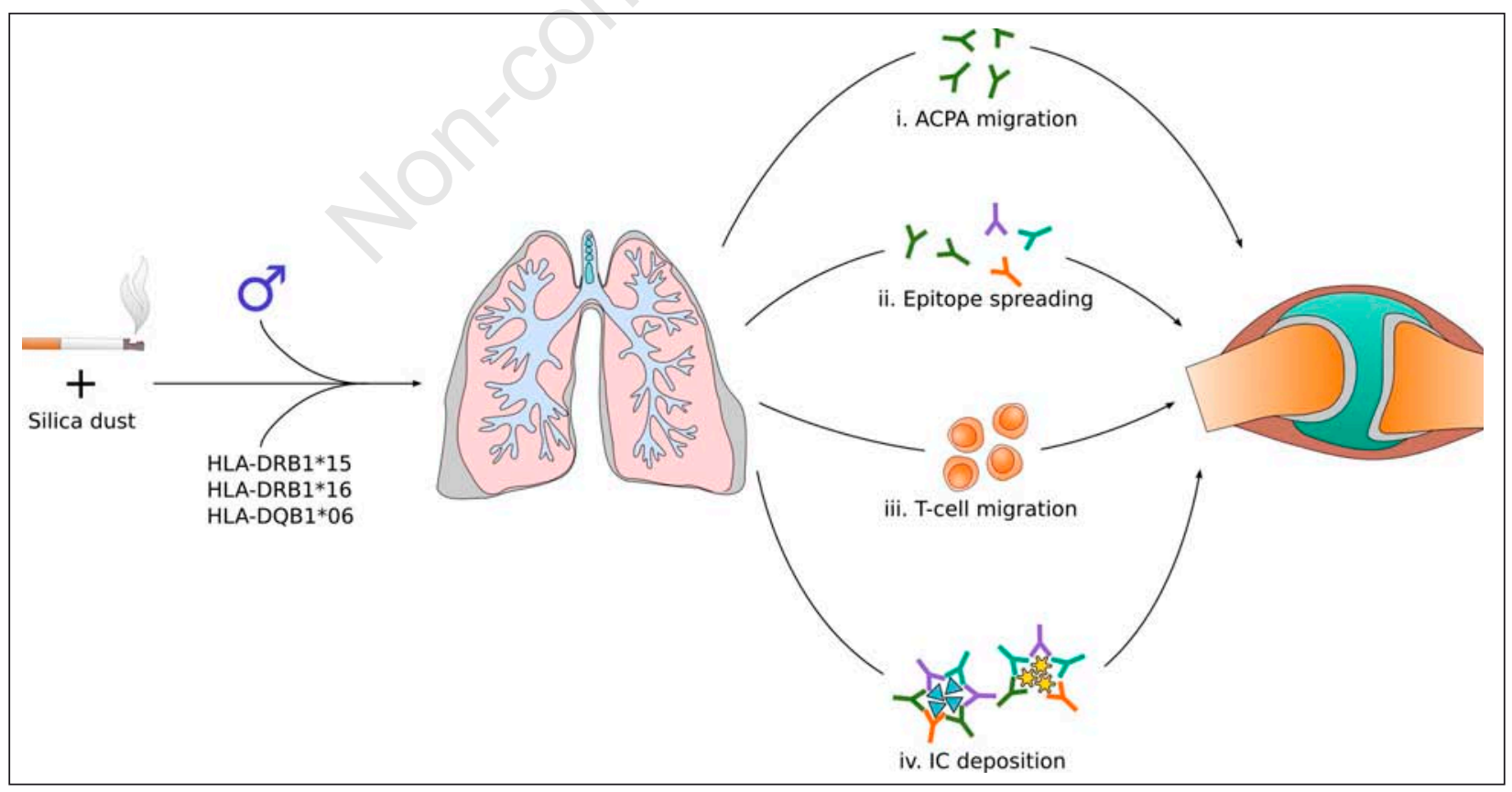

Figure 1 - Possible mechanisms suggested to explain the spreading of inflammation from lungs to joints in rheumatoid arthritis. 
quently triggering and orchestrating the inflammatory process.

4. Immune complex deposition: the presence of immune complexes in the joints of patients with RA and their proinflammatory role is well established. Among these, ACPA immune complexes are able to induce TNF- $\alpha$ production by macrophages. It could therefore be speculated that such immune complexes could originate in the lung and subsequently reach synovial tissues through the blood stream, thereby triggering arthritis (44).

\section{BEYOND CITRULLINATION}

Despite the high specificity of ACPA for the diagnosis of RA and the association of serum ACPA-positivity with RA-ILD, a large proportion of patients with RA and RA-ILD do not display such antibodies in the serum. This finding is probably due to multiple causes. First of all, commercially available diagnostic kits for the detection of ACPA include a citrullinated cyclic peptide (CCP) which is used to bind and detect ACPA directed towards a certain amount of epitopes. Nevertheless, a wider spectrum of ACPA can be detected using more advanced kits with higher sensitivity which will increasingly become available for diagnostic purposes. It should be therefore kept in mind that putative ACPA-negative RA patients may actually have such antibodies in the serum that unfortunately cannot yet be routinely detected.

Despite such observation, it cannot be ruled out that other non-citrullinated molecules may be involved in RA and ILD-RA pathogenesis, consequently or independently of autoimmunity. Among these, new autoantibody systems have been discovered which appear to be involved in the pathogenesis of RA, such as anti-carbamylated protein (anti-CarP) antibodies. Carbamylation, like citrullination, is a post-translational modification in which lysine residues are converted to homocitrulline residues. Just as with ACPA, anti-CarP antibodies appear in the blood of RA patients several years before disease onset and, despite similari- ties shared with ACPA, they represent two independent systems, as suggested by the findings of patients presenting exclusively anti-CarP antibodies and not ACPA (45). The same observation can be applied to anti-malondialdehyde-acetaldehyde (antiMAA) antibodies which have been detected in a significant percentage of ACPA-negative RA patients. MAA adducts, currently under investigation for their possible role in cardiovascular diseases, are the result of lipid peroxydation and have been shown to co-localize with citrullinated antigens in the synovia of patients with RA compared to those with osteoarthritis (OA) $(45,46)$.

Radiological and histopathological features of lungs in ILD-RA clearly support an intense tissue remodeling, very likely due to local inflammation. Therefore, it should be intriguing to investigate the relationship between inflammation and tissue remodeling. In this setting, IL-4 is able to stimulate proliferation and differentiation of fibroblasts and epithelial cells contributing to tissue remodeling, but is also involved in promoting the shift from Th1 to Th2 immune response, with potential protective effects. A study reported that patients with RA-ILD display higher BAL levels of IL-4 compared to those without lung involvement. This observation is interesting and may link IL-4 to tissue remodeling. However, since it comes from a pilot study, needs to be confirmed by other studies (47).

Tissue remodeling is a key feature of both IPF and RA-ILD and various authors have investigated its mechanisms. In this setting, Chen et al. (26) demonstrated that serum levels of MMP-7 correlate with the severity of RA-ILD in patients with either fully blown or subclinical disease. MMP-7 is also known to be involved in IPF, hence this may be a shared pathway in the pathogenesis of both conditions. Similarly, other authors have recently identified a dysfunction of NK cells in both IPF and RA-NSIP, suggesting that this cell type may be a leading player in these two diseases (48). Taking these observations together, it may be speculated that the distinct pathological processes causing IPF and the various 
subtypes of RA-ILD may share pathogenic mechanisms culminating in a common final step leading to tissue remodeling. These pathways may therefore represent potential targets for the treatment of multiple diseases.

Nevertheless, the subtypes of RA-ILD can indeed be distinguished not only by their different clinical and radiologic features, but also by their pathogenic aspects. Various molecules and proteins have been found to be differentially expressed in RAILD subtypes. Lower BAL TNF- $\alpha$ concentrations have been described in patients with RA-UIP compared to other subtypes. Furthermore, an increased toll-like receptor (TLR)-2 expression on monocytes from RA-OP, RA-UIP and RA-NSIP patients compared to healthy controls, and an overexpression of the pattern recognition triggering receptor expressed on myeloid cells (TREM)- 1 on neutrophils and monocytes of patients with RA-OP compared to healthy controls have been reported (49). An interesting area of future research could be an investigation of the role of telomeres and telomerase activity in cells implicated in RA-ILD pathogenesis. Their implication in IPF is well established (50) and very little is known about their role in RA and other autoimmune diseases. The results of the few studies available seem to show an actual impairment of telomeres biology. However, the results are conflicting and not strictly comparable due to differences in the study design and techniques employed $(51,52)$.

Clarification of similarities and differences in the pathogenic mechanisms underlying different types of RA-ILD may allow the identification of surrogate disease biomarkers to help clinicians in the early classification and stratification of patients with RA-ILD. The final goal may be to predict disease evolution and therefore establish tailored follow-up and treatment strategies.

\section{CONCLUSIONS}

Considering our current knowledge regarding RA pathogenesis, it is reasonable to speculate that the lung represents one of the possible sites of autoimmunity initiation eventually leading to the development of RA and RA-ILD. Unfortunately, however, the body of evidence does not allow us to draw definitive conclusions and several aspects need to be clarified. However, this line of investigation appears to be worth pursuing, as the proof that RA is a systemic disease from the beginning, even in the absence of evident extra-articular involvement, would help clinicians to tailor and optimize preventive, diagnostic and therapeutic strategies.

\section{Competing interests: nothing to disclose}

\section{REFERENCES}

1. Zou Y-Q, Li Y-S, Ding X-N, Ying Z-H. The clinical significance of HRCT in evaluation of patients with rheumatoid arthritis-associated interstitial lung disease: a report from China. Rheumatol Int. 2012; 32: 669-73.

2. Berthelot JM, Bataille R, Maugars Y, Prost A. Rheumatoid arthritis as a bone marrow disorder. Semin Arthritis Rheum. 1996; 26: 505-14.

3. Giles JT, Danoff SK, Sokolove J, et al. Association of fine specificity and repertoire expansion of anticitrullinated peptide antibodies with rheumatoid arthritis associated interstitial lung disease. Ann Rheum Dis. 2014; 73: 1487- 94.

4. Giles JT, Darrah E, Danoff S, et al. Association of cross-reactive antibodies targeting peptidylarginine deiminase 3 and 4 with rheumatoid arthritis-associated interstitial lung disease. PLoS One. 2014; 9: e98794.

5. Yin Y, Liang D, Zhao L, et al. Anti-cyclic citrullinated peptide antibody is associated with interstitial lung disease in patients with rheumatoid arthritis. PLoS One. 2014; 9: e92449.

6. Cavagna L, Monti S, Grosso V, et al. The multifaceted aspects of interstitial lung disease in rheumatoid arthritis. BioMed Res Int. 2013; 2013: 759760.

7. Lake F, Proudman S. Rheumatoid arthritis and lung disease: from mechanisms to a practical approach. Semin Respir Crit Care Med. 2014; 35: 222-38.

8. Hallowell RW, Horton MR. Interstitial lung disease in patients with rheumatoid arthritis: spontaneous and drug induced. Drugs. 2014; 74: 443-50.

9. Kelly CA, Saravanan V, Nisar M, et al. Rheumatoid arthritis-related interstitial lung disease: associations, prognostic factors and physiological and radiological characteristicsa large multicentre UK study. Rheumatol Oxf Engl. 2014; 53: 1676-82. 
10. Chen J, Shi Y, Wang X, et al. Asymptomatic preclinical rheumatoid arthritis-associated interstitial lung disease. Clin Dev Immunol. 2013; 2013: 406927.

11. Gochuico BR, Avila NA, Chow CK, et al. Progressive preclinical interstitial lung disease in rheumatoid arthritis. Arch Intern Med. 2008; 168: 159-66.

12. Assayag D, Lubin M, Lee JS, et al. Predictors of mortality in rheumatoid arthritis-related interstitial lung disease. Respirol Carlton Vic. 2014; 19: 493-500.

13. Bongartz T, Nannini C, Medina-Velasquez YF, et al. Incidence and mortality of interstitial lung disease in rheumatoid arthritis: a population-based study. Arthritis Rheum. 2010; 62: 1583-91.

14. Mori S, Cho I, Koga Y, Sugimoto M. Comparison of pulmonary abnormalities on high-resolution computed tomography in patients with early versus longstanding rheumatoid arthritis. J Rheumatol. 2008; 35: 1513-21.

15. Gregersen PK, Silver J, Winchester RJ. The shared epitope hypothesis. An approach to understanding the molecular genetics of susceptibility to rheumatoid arthritis. Arthritis Rheum. 1987; 30: 1205-13.

16. Furukawa H, Oka S, Shimada K, et al. Association of human leukocyte antigen with interstitial lung disease in rheumatoid arthritis: a protective role for shared epitope. PLoS One. 2012; 7: e33133.

17. Aubart F, Crestani B, Nicaise-Roland P, et al. High levels of anti-cyclic citrullinated peptide autoantibodies are associated with co-occurrence of pulmonary diseases with rheumatoid arthritis. J Rheumatol. 2011; 38: 979-82.

18. Wang J-X, Du C-G. A retrospective study of clinical characteristics of interstitial lung disease associated with rheumatoid arthritis in Chinese patients. Med Sci Monit Int Med J Exp Clin Res. 2015; 21: 708-15.

19. Yahya A, Bengtsson C, Larsson P, et al. Silica exposure is associated with an increased risk of developing ACPA-positive rheumatoid arthritis in an Asian population: evidence from the Malaysian MyEIRA case-control study. Mod Rheumatol Jpn Rheum Assoc. 2014; 24 : 271-4.

20. Catrina AI, Deane KD, Scher JU. Gene, environment, microbiome and mucosal immune tolerance in rheumatoid arthritis. Rheumatol Oxf Engl. 2016; 55: 391-402.

21. Roubille C, Haraoui B. Interstitial lung diseases induced or exacerbated by DMARDS and biologic agents in rheumatoid arthritis: a systematic literature review. Semin Arthritis Rheum. 2014; 43: 613-26.

22. Alexiou I, Germenis A, Koutroumpas A, et al. Anti-cyclic citrullinated peptide-2 (CCP2) autoantibodies and extra-articular manifestations in Greek patients with rheumatoid arthritis. Clin Rheumatol. 2008; 27: 511-3.

23. Zhu J, Zhou Y, Chen X, Li J. A metaanalysis of the increased risk of rheumatoid arthritisrelated pulmonary disease as a result of serum anticitrullinated protein antibody positivity. J Rheumatol. 2014; 41: 1282-9.

24. Reynisdottir G, Karimi R, Joshua V, et al. Structural changes and antibody enrichment in the lungs are early features of anti-citrullinated protein antibody-positive rheumatoid arthritis. Arthritis Rheumatol Hoboken NJ. 2014; 66: 31-9.

25. Inui N, Enomoto N, Suda T, et al. Anti-cyclic citrullinated peptide antibodies in lung diseases associated with rheumatoid arthritis. Clin Biochem. 2008; 41: 1074-7.

26. Chen J, Doyle TJ, Liu Y, et al. Biomarkers of rheumatoid arthritis-associated interstitial lung disease. Arthritis Rheumatol Hoboken NJ. 2015; 67: 28-38.

27. Alunno A, Caneparo V, Bistoni O, et al. The circulating interferon-inducible protein IFI16 correlates with clinical and serological features in rheumatoid arthritis. Arthritis Care Res. 2015 [Epub ahead of print].

28. Harlow L, Rosas IO, Gochuico BR, et al. Identification of citrullinated hsp90 isoforms as novel autoantigens in rheumatoid arthritisassociated interstitial lung disease. Arthritis Rheum. 2013; 65: 869-79.

29. Harlow L, Gochuico BR, Rosas IO, et al. Anticitrullinated heat shock protein 90 antibodies identified in bronchoalveolar lavage fluid are a marker of lung-specific immune responses. Clin Immunol Orlando Fla. 2014; 155: 60-70.

30. Demoruelle MK, Weisman MH, Simonian PL, et al. Brief report: airways abnormalities and rheumatoid arthritis-related autoantibodies in subjects without arthritis: early injury or initiating site of autoimmunity? Arthritis Rheum. 2012; 64: 1756-61.

31. Demoruelle MK, Deane KD, Holers VM. When and where does inflammation begin in rheumatoid arthritis? Curr Opin Rheumatol. 2014; 26: 64-71.

32. Perry E, Kelly C, Eggleton P, et al. The lung in ACPA-positive rheumatoid arthritis: an initiating site of injury? Rheumatol Oxf Engl. 2014; 53: $1940-50$.

33. Willis VC, Demoruelle MK, Derber LA, et al. Sputum autoantibodies in patients with established rheumatoid arthritis and subjects at risk of future clinically apparent disease. Arthritis Rheum. 2013; 65: 2545-54.

34. Makrygiannakis D, Hermansson M, Ulfgren A-K, et al. Smoking increases peptidylarginine deiminase 2 enzyme expression in human lungs and increases citrullination in BAL cells. Ann Rheum Dis. 2008; 67: 1488-92.

35. Källberg H, Ding B, Padyukov L, et al. Smoking is a major preventable risk factor for rheu- 
matoid arthritis: estimations of risks after various exposures to cigarette smoke. Ann Rheum Dis. 2011; 70: 508-11.

36. Linn-Rasker SP, van der Helm-van Mil AHM, van Gaalen FA, et al. Smoking is a risk factor for anti-CCP antibodies only in rheumatoid arthritis patients who carry HLA-DRB1 shared epitope alleles. Ann Rheum Dis. 2006; 65: 366-71.

37. Lugli EB, Correia RESM, Fischer R, et al. Expression of citrulline and homocitrulline residues in the lungs of non-smokers and smokers: implications for autoimmunity in rheumatoid arthritis. Arthritis Res Ther. 2015; 17: 9.

38. Rangel-Moreno J, Hartson L, Navarro C, et al. Inducible bronchus-associated lymphoid tissue (iBALT) in patients with pulmonary complications of rheumatoid arthritis. J Clin Invest. 2006; 116: 3183-94.

39. Janssen KMJ, de Smit MJ, Brouwer E, et al. Rheumatoid arthritis-associated autoantibodies in non-rheumatoid arthritis patients with mucosal inflammation: a case-control study. Arthritis Res Ther. 2015; 17: 174.

40. Perry E, Stenton C, Kelly C, et al. RA autoantibodies as predictors of rheumatoid arthritis in non-cystic fibrosis bronchiectasis patients. Eur Respir J. 2014; 44: 1082-5.

41. Wegner N, Wait R, Sroka A, et al. Peptidylarginine deiminase from Porphyromonas gingivalis citrullinates human fibrinogen and $\alpha$-enolase: implications for autoimmunity in rheumatoid arthritis. Arthritis Rheum. 2010; 62: 2662-72.

42. Ytterberg AJ, Joshua V, Reynisdottir G, et al. Shared immunological targets in the lungs and joints of patients with rheumatoid arthritis: identification and validation. Ann Rheum Dis. 2015; 74: 1772-7.

43. Sokolove J, Bromberg R, Deane KD, et al. Autoantibody epitope spreading in the pre-clini- cal phase predicts progression to rheumatoid arthritis. PLoS One. 2012; 7: e35296.

44. Laurent L, Anquetil F, Clavel C, et al. IgM rheumatoid factor amplifies the inflammatory response of macrophages induced by the rheumatoid arthritis-specific immune complexes containing anticitrullinated protein antibodies. Ann Rheum Dis. 2015; 74: 1425-31.

45. Shi J, van de Stadt LA, Levarht EWN, et al. Anti-carbamylated protein (anti-CarP) antibodies precede the onset of rheumatoid arthritis. Ann Rheum Dis. 2014; 73: 780-3.

46. Thiele GM, Duryee MJ, Anderson DR, et al. Malondialdehyde-acetaldehyde adducts and anti-malondialdehyde-acetaldehyde antibodies in rheumatoid arthritis. Arthritis Rheumatol Hoboken NJ. 2015; 67: 645-55.

47. Shen H, Xia L, Lu J. Interleukin-4 in rheumatoid arthritis patients with interstitial lung disease: a pilot study. Indian J Med Res. 2013; 138: 919-21.

48. Craig VJ, Zhang L, Hagood JS, Owen CA. Matrix metalloproteinases as therapeutic targets for idiopathic pulmonary fibrosis. Am J Respir Cell Mol Biol. 2015; 53: 585-600.

49. Papanikolaou IC, Boki KA, GiamarellosBourboulis EJ, et al. Innate immunity alterations in idiopathic interstitial pneumonias and rheumatoid arthritis-associated interstitial lung diseases. Immunol Lett. 2015; 163: 179-86.

50. Spagnolo P, Grunewald J, du Bois RM. Genetic determinants of pulmonary fibrosis: evolving concepts. Lancet Respir Med. 2014; 2: 416-28.

51. Fujii H, Shao L, Colmegna I, et al. Telomerase insufficiency in rheumatoid arthritis. Proc Natl Acad Sci USA. 2009; 106: 4360-5.

52. Tamayo M, Mosquera A, Rego JI, et al. Differing patterns of peripheral blood leukocyte telomere length in rheumatologic diseases. Mutat Res. 2010; 683: 68-73. 